\title{
HMGA1-mediated miR-671-5p targets APC to promote metastasis of clear cell renal cell carcinoma through Wnt signaling
}

\author{
X. G. CHI ${ }^{1, *}$, X. X. MENG ${ }^{2, *}$, D. L. DING ${ }^{1}$, X. H. XUAN ${ }^{1}$, Y. Z. CHEN ${ }^{1}$, Q. CAI ${ }^{1}$, A. WANG ${ }^{3, *}$ \\ ${ }^{1}$ The Affiliated Xiaolan Hospital of Southern Medical University, No. 65 Middle Section of Xiaolan Jucheng Avenue, Zhongshan 528415, China; \\ ${ }^{2}$ Guangzhou TCM Hospital, No. 16 Zhuji Road, Liwan District, Guangzhou 510515, China; ${ }^{3}$ Guangdong Second Provincial General Hospital, \\ Courtyard 466 Xingang Middle Road, Haizhu District, Guangzhou 510000, China
}

${ }^{*}$ Correspondence: wa510000@163.com

${ }^{*}$ Contributed equally to this work.

Received February 17, 2019 / Accepted May 29, 2019

\begin{abstract}
This study aimed to investigate the effect of miR-671-5p on metastasis of clear cell renal cell carcinoma (ccRCC) and underlying mechanism involved. The migration and invasion of ccRCC cells were determined by transwell and boyden assays in vitro and in vivo. Genes mRNA and protein expression were detected by quantitative polymerase chain reaction (qPCR) and western blot analysis, respectively. The target gene of miRNA was confirmed by luciferase reporter assays. Transcriptional regulation of miRNA by transcription factor was detected by chromatin immunoprecipitation assay (ChIP). The expressions of miRNA in clinical specimens were detected by in situ hybridization (ISH). miR-671-5p promoted migration and invasion of ccRCC in vivo and in vitro. Moreover, miR-671-5p directly targeted APC to activate Wnt signaling, thus inducing the epithelial-mesenchymal transition (EMT) in ccRCC. Intriguingly, miR-671-5p expression was transcriptionally enhanced by HMGA1. Consistently, bioinformatics analysis suggested that HMGA1 was positively correlated with miR-671 expression; however, miR-671 was negatively correlated with APC. In situ hybridization analysis showed that miR-671-5p was upregulated in ccRCC compared with paracarcinoma and correlated with poor prognosis of ccRCC patients. In addition, univariate and multivariate analysis indicated that miR-671-5p expression was an independent prognostic factor for overall survival in ccRCC patients. Our data suggest that miR-671-5p is a tumor enhancer in regulating of ccRCC metastasis, and miR-671-5p may be utilized as a factor for the clinical diagnosis and prognosis of ccRCC.
\end{abstract}

Key words: miR-671-5p, APC, HMGA1, clear cell renal cell carcinoma

Renal cell cancer is becoming more commonly diagnosed worldwide in both men and women [1]. Clear cell renal cell carcinoma (ccRCC) is the most common histological subtype of renal cell carcinoma and accounts for most cancer-related deaths [2]. Cancer metastasis confers the poor prognosis of ccRCC patients. Therefore, it is meaningful to reveal the molecular mechanisms underlying ccRCC metastasis and uncover novel indicators of survival and candidate therapeutic targets. Epithelial-mesenchymal transition (EMT) that acquires fibroblastoid properties and reduces intercellular adhesion in epithelial cells has been regarded as a crucial process in cancer metastasis $[3,4]$. The characteristic shifts during EMT process are upregulation of mesenchymal markers (N-cadherin, Vimentin) and downregulation of epithelial markers (E-cadherin) [5].

Small noncoding microRNAs (miRNAs) that are differentially expressed in normal tissues and cancers contribute to cancer development and progression $[6,7]$. miRNAs can function as oncomiRs through enforcing cancer metastasis in ccRCC $[8,9]$. Therefore, strategies that target oncomiRs may be alternative manners to overcome ccRCC metastasis. However, the role of miR-671-5p in ccRCC remains unknown.

Here, we demonstrated that miR-671-5p acts as an oncomiR in ccRCC in vivo and in vitro. miR-671-5p was positively correlated with poor patient prognosis. Mechanistic analysis indicated that miR-671-5p directly targeted APC to activate Wnt signaling and facilitated the EMT process, thus promoting ccRCC metastasis. Moreover, miR-671-5p-induced signals are enhanced by the oncogene HMGA1.

\section{Materials and methods}

Cell culture. The human ccRCC cell lines 786-O, CAKI-1 and the renal epithelial cell line HK-2 were purchased from 
the Shanghai Cell Bank of Chinese Academy of Sciences. 786-O and CAKI- 1 were cultured in RPMI 1640 medium, and HK-2 was cultured in DMEM/F12 medium supplemented with $10 \%(\mathrm{v} / \mathrm{v})$ fetal bovine serum (FBS) in a humidified atmosphere of $5 \% \mathrm{CO}_{2}$ at $37^{\circ} \mathrm{C}$ in the incubator.

Lentivirus infection. Lentivirus encoding shRNA targeting miR-671 (sh-miR-671), miR-671 sequence (ov-miR-671) and their negative control sequences (NC) were constructed by GeneChem, Shanghai, China. Lentivirus vectors were transfected into ccRCC cells using Polybrene reagent (Sigma-Aldrich, St. Louis, MO, USA).

Transient transfection with siRNAs, plasmids or miR-671-5p mimics, inhibitor. Cells were seeded onto 6-well plates. Specific siRNA, plasmid, mimics or inhibitor were then transfected into ccRCC cells using TurboFect siRNA Transfection Reagent following the manufacturer's protocol. Cells were collected $48-72 \mathrm{~h}$ after transfection for subsequent experiments.

RNA isolation, reverse transcription, and QPCR. After RNA isolation, reverse transcription was performed using reverse transcription reagents (Thermo Scientific, Waltham, MA, USA). QPCR was conducted using a SYBR Premix Ex TaqTM II kit (TaKaRa Bio, Inc., Shiga, Japan) according to the manufacturer's instructions.

Transwell and Boyden assays. For transwell assays, a total of $1 \times 10^{5}$ cells were seeded into transwell chambers. Twelve hours later, the migrated cells were fixed with methanol, stained with Giemsa solution, and photographed under a microscope. The boyden chambers were applied for invasion assays, the procedures of boyden assays were similar to transwell assays.

In vivo metastasis assay in nude mice. The protocols for the animal study were approved by the Institutional Animal Ethics Committee Experimental Animal Center of Southern Medical University, China (No. 2018PSl39K). Four weeks old male BALB/c nude mice were purchased from SLAC Laboratory Animal Co. Ltd., Shanghai, China. For in vivo metastasis assay, $50 \mu \mathrm{l}$ of a total of $1 \times 10^{6}$ cells/mouse were intravenously injected into the mice. All mice were sacrificed 6 weeks after cells inoculation. The mice were maintained in a barrier facility and fed with an autoclaved laboratory rodent diet.

Western blot analysis. Cell lysates were collected in lysis buffer and the protein concentration was detected using a BCA protein assay kit (Thermo Scientific, Waltham, MA, USA). Total proteins were separated by $8-10 \%$ SDS-PAGE and transferred onto polyvinyldifluoride (PVDF) membranes. The membranes were then incubated with the primary antibodies overnight at $4{ }^{\circ} \mathrm{C}$ and immunoprobed with the secondary antibody for $1 \mathrm{~h}$ at room temperature. The proteins signals were measured by an enhanced chemiluminescence reagent (Thermo Scientific, Waltham, MA, USA), and images were captured using a ChemiDocTM CRS + Molecular Imager (Bio-Rad).

Luciferase reporter assay. Fragments of the APC 3'UTR were amplified by PCR and cloned into psiCHECK- 2 vectors (named wt). Site-directed mutagenesis of miR-671-5p binding site in the APC 3'UTR was performed using GeneTailor Site-Directed Mutagenesis System (Invitrogen, named $\mathrm{mt}$ ). For reporter assays, wild-type (wt), mutant (mt) or control psiCHECK-2 vectors were co-transfected into cells with miR-671-5p mimics or inhibitor. Luciferase activity was measured at $48 \mathrm{~h}$ after transfection by Dual-Luciferase Reporter Assay System (Promega Corporation, Madison, WI, USA).

Chromatin immunoprecipitation assay. Chromatin immunoprecipitation assays (ChIP) were performed using a ChIP assay kit (Thermo Scientific, Waltham, MA, USA) following the manufacturer's instructions. Briefly, chromatin was crosslinked, isolated, and digested with micrococcal nuclease to obtain DNA fragments. The anti-HMGA1 was adopted for immunoprecipitation overnight at $4{ }^{\circ} \mathrm{C}$. After elution and purification, the recovered DNA fragments were used for QPCR analysis.

Bioinformatics analysis. miRNA and mRNA expression profiles of kidney renal clear cell carcinoma were analyzed based on TCGA KIRC database. The latest miRNA-Seq and mRNA-Seq data of KIRC were downloaded and subjected for differential expression and survival analysis. The $\mathrm{R}$ program was used for differential expression and survival analysis. GSEA java program was applied for gene set enrichment analysis.

Patient tissues. Ninety primary ccRCC tissues and 90 paracarcinoma tissues were purchased from Shanghai Outdo Biotech Co. Ltd (Shanghai, China). All specimens had a confirmed pathological diagnosis. Approval from the Ethics Committee of the People's Hospital of Maoming was obtained before the use of these clinical materials for research.

In situ hybridization (ISH). In situ hybridization was conducted on paraffin-embedded sections. After deparaffinization and rehydration, sections were incubated with proteinase $\mathrm{K}$ and then were rinsed, fixed and prehybridized. Slides were hybridized with digoxigenin (DIG)-labeled locked nucleic acid (LNA) probes and immunoprobed with anti-DIG-HRP Fab fragments. Signals were visualized by adding 3,3'-diaminobenzidine (DAB) substrate, and the staining intensity was evaluated and scored.

Evaluation and scoring. The ISH sections were evaluated and scored by two pathologists blindly. Staining intensity was estimated as 0 (no staining), 1 (weakly positive staining), 2 (moderately positive staining) and 3 (strong staining). The percentages were scored on a scale of 0 to 4 , as follows: $0(0 \%)$, $1(\leq 25 \%), 2$ (26-50\%), 3 (51-75\%), and $4(76-100 \%)$. The final staining scores were obtained through multiplying the intensity score by the percentage score. Samples with staining scores $0-8$ were regarded as low expression, and those with staining scores 9-12 were regarded as high expression.

Statistical analysis. All data were analyzed by SPSS 21.0 software. The data are expressed as the mean \pm SD from at least three independent experiments. Statistical significance was measured by Student's two-tailed $t$ test for two groups, 
one-way ANOVA for multiple groups. The skewed data were analyzed by Wilcoxon rank sum test. Correlation between gene expression and clinicopathological features were analyzed by Chi-square test. Log-rank tests were conducted on Kaplan-Meier survival curves to measure any significant relationship between gene expression and overall patient survival. Univariate and multivariate survival analysis were performed using the Cox proportional hazards regression model. The hazard ratio (HR) and corresponding 95\% confidence intervals $(95 \% \mathrm{CI})$ were calculated for each factor. All tests were two-sided, and a p-value of $<0.05$ was considered statistically significant. ${ }^{*} \mathrm{p}<0.05,{ }^{\star *} \mathrm{p}<0.01$ and ${ }^{\star * *} \mathrm{p}<0.001$.

\section{Results}

miR-671-5p is upregulated in ccRCC and correlates with poor patients' prognosis. To clarify the role of miRNAs in ccRCC, we focused on TCGA data mining to identify miRNAs that are significantly correlated with overall survival of ccRCC patients. Therein, miR-671, which has not been studied in ccRCC, was a potential oncomiR that conferred poor patients' prognosis in ccRCC (Figure 1A). Furthermore, miR-671 expression was lower in early TNM stage compared with late TNM stage (Stage I-II vs. III-IV, T1-2 vs. T3-4, and M0 vs. M1) (Figures 1B-D). Gene set enrichment analysis (GSEA) based on TCGA ccRCC dataset revealed that miR-671 was positively involved in regulating EMT process (Figure 1E). Moreover, we further found that miR-671-5p (not miR-671-3p) was elevated in ccRCC and was positively associated with poor prognosis of ccRCC patients in OncomiR database $(\mathrm{p}<0.001)$ (www.oncomir. org). Then, we performed in situ hybridization (ISH) in 90 ccRCC and 90 paracarcinoma tissues. Consistently, miR-671-5p expression was elevated in ccRCC compared with paracarcinoma tissues $(\mathrm{p}=0.004)$ (Figure 1F, Table 1). The correlation analysis showed that miR-671-5p expression was positively associated with American Joint Committee on Cancer (AJCC) stage, but not with other parameters (Table 2). The survival analysis further suggested that ccRCC patients with high miR-671-5p expression predicted poorer overall survival than those with low miR-671-5p expression (Figure $1 \mathrm{G}$ ). In addition, univariate and multivariate analysis indicated that miR-671-5p expression was an independent prognostic factor for overall survival in ccRCC patients $(\mathrm{HR}=0.677,95 \%$ CI $0.493-0.968, \mathrm{p}=0.047)$ (Table 3).

Table 1. miR-671-5p expression in ccRCC tissues and paracarcinoma tissues.

\begin{tabular}{lcccc}
\hline \multirow{2}{*}{ Group } & Cases & \multicolumn{2}{c}{ miR-671-5p expression } & \\
\cline { 3 - 4 } & $(\mathbf{n})$ & Low & High & \\
\hline clear cell renal cell carcinoma & 90 & $46(51.1 \%)$ & $44(48.9 \%)$ & \multirow{2}{*}{0.004} \\
paracarcinoma & 90 & $27(30.0 \%)$ & $63(70.0 \%)$ & \\
\hline
\end{tabular}

A

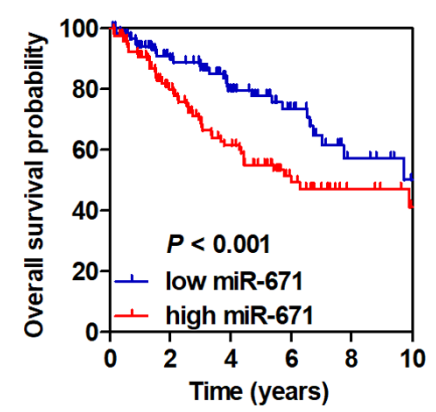

E

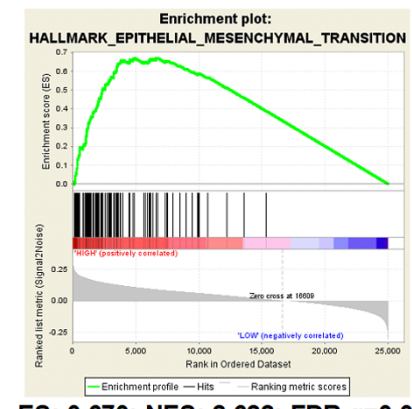

ES: $0.670 ;$ NES: $2.622 ;$ FDR $q=0.0$
B

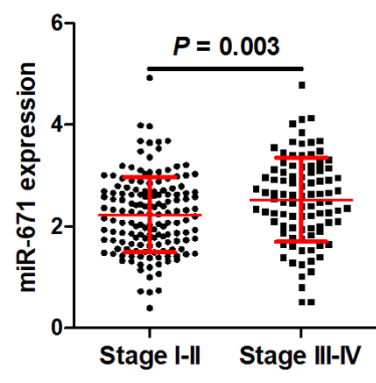

$\mathbf{F}$

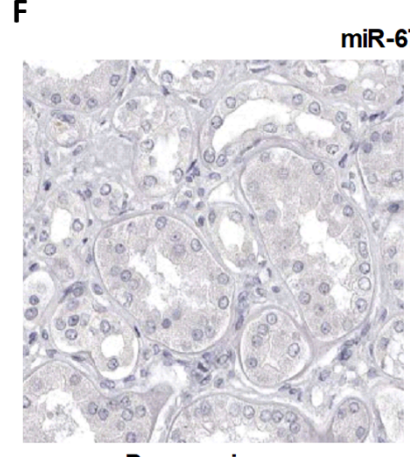

Paracarcinoma
C

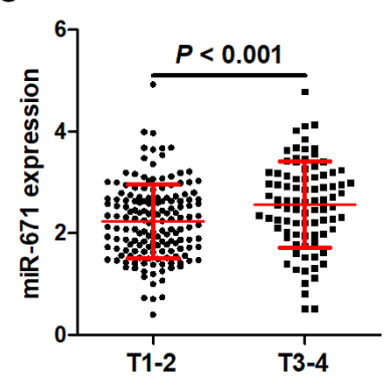

$\mathbf{G}$

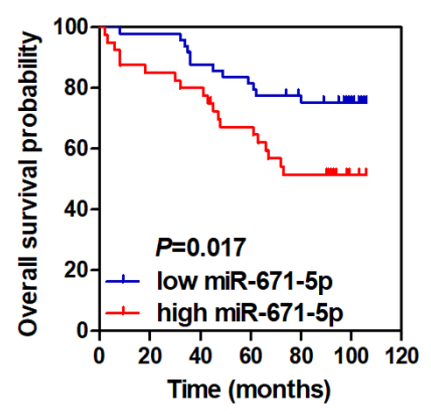

Figure 1. miR-671-5p is upregulated in ccRCC and correlates with poor patients' prognosis. A) Kaplan-Meier survival analysis of ccRCC patients with low and high miR-671 expression based on TCGA KIRC miRNA-seq data. B-D) Differential expression of miR-671 in TNM stage (Stage I-II vs. III-IV, T1-2 vs. T3-4, and M0 vs. M1). E) GSEA of miR-671 regulated signaling based on the TCGA database. F) ISH detection of miR-671-5p expression in ccRCC tissues and paracarcinoma tissues. G) Kaplan-Meier survival analysis of ccRCC patients with low and high miR-671-5p expression detected by ISH. 
miR-671-5p promotes metastasis of ccRCC cells in vivo and in vitro. To clearly elucidate the biological function of miR-671-5p in ccRCC, we detected the expression of miR-671-5p in ccRCC cell lines and the renal epithelial cell line. QPCR assays revealed that miR-671-5p was upregulated in 786-O and CAKI-1 compared with HK-2, while miR-671-5p expression in CAKI-1 cells with high metastatic potential was higher than that in 786-O cells with low metastatic potential (Figure 2A). To further clarify the effect of miR-671-5p on ccRCC, we established miR-671-5poverexpressed 786-O cells or miR-671-5p-silenced CAKI-1 cells via mimics, inhibitor or lentivirus vectors (Figure $2 \mathrm{~B}$ ). miR-671-5p upregulation enhanced migration and invasion of 786-O cells, however, miR-671-5p knockdown impaired migration and invasion of CAKI-1 cells (Figures 2C, 2D). In the xenograft models, mice intravenously injected with
miR-671-overexpressed 786-O cells exhibited an increase in the number of metastatic lung nodules and the incidence of lung metastasis compared with mice injected with the control cells, while the opposite results were obtained in mice injected with miR-671-silenced CAKI-1 cells and the control cells (Figure 2E, Table 4).

miR-671-5p directly targets APC and modulates APC-mediated Wnt signaling and EMT in ccRCC. APC was predicted to be a direct target of miR-671-5p by Targetscan, miRWalk and RNAhybrid database (Figure 3A). Upregulation of miR-671-5p downregulated APC protein and mRNA levels in 786-O cells, while knockdown of miR-671-5p elevated APC expression in CAKI-1 cells (Figures 3B, 3C). Then, APC was confirmed as a target of miR-671-5p by luciferase reporter assay. Introduction of miR-671-5p inhibitor enhanced the luciferase activity of the wild type APC reporter,

Table 2. Correlations between miR-671-5p expression and the clinicopathological features of ccRCC patients.

\begin{tabular}{|c|c|c|c|c|}
\hline \multirow{2}{*}{ Characteristics } & \multirow{2}{*}{ Total } & \multicolumn{2}{|c|}{ miR-671-5p expression } & \multirow{2}{*}{ p-value } \\
\hline & & Low, n (\%) & High, n (\%) & \\
\hline Age (years) & & 46 & 44 & \\
\hline$\leq$ Median & 46 & $26(56.5 \%)$ & $20(43.5 \%)$ & 0.294 \\
\hline >Median & 44 & $20(45.5 \%)$ & $24(54.5 \%)$ & \\
\hline \multicolumn{5}{|l|}{ Gender } \\
\hline Male & 51 & $25(49.0 \%)$ & $26(51.0 \%)$ & 0.650 \\
\hline Female & 39 & $21(53.8 \%)$ & $18(46.2 \%)$ & \\
\hline \multicolumn{5}{|l|}{ AJCC stage } \\
\hline I-II & 77 & $43(55.8 \%)$ & $34(44.2 \%)$ & 0.029 \\
\hline III-IV & 13 & $3(23.1 \%)$ & $10(76.9 \%)$ & \\
\hline \multicolumn{5}{|l|}{ Fuhrman grade } \\
\hline I-II & 75 & $40(53.5 \%)$ & $35(46.7 \%)$ & 0.346 \\
\hline III-IV & 15 & $6(40.0 \%)$ & $9(60.0 \%)$ & \\
\hline
\end{tabular}

Table 3. Univariate and multivariate analysis of clinicopathological variables of ccRCC patients.

\begin{tabular}{|c|c|c|c|c|c|c|}
\hline \multirow{3}{*}{ Clinical parameters } & \multicolumn{6}{|c|}{ Overall survival } \\
\hline & \multicolumn{3}{|c|}{ Univariate analysis } & \multicolumn{3}{|c|}{ Multivariate analysis } \\
\hline & HR & 95\% CI & p-value & HR & 95\% CI & p-value \\
\hline $\begin{array}{l}\text { miR-671-5p expression } \\
\text { Low } \\
\text { High }\end{array}$ & 0.416 & $(0.203-0.856)$ & 0.017 & 0.677 & $(0.493-0.968)$ & 0.047 \\
\hline $\begin{array}{l}\text { Age (years) } \\
\leq \text { Median } \\
>\text { Median }\end{array}$ & 0.457 & $(0.225-0.927)$ & 0.030 & 0.736 & $(0.404-1.698)$ & 0.614 \\
\hline $\begin{array}{l}\text { Gender } \\
\text { Male } \\
\text { Female }\end{array}$ & 0.711 & $(0.346-1.460)$ & 0.352 & & & \\
\hline $\begin{array}{l}\text { AJCC stage } \\
\quad \text { I-II } \\
\text { III-IV }\end{array}$ & 0.090 & $(0.031-0.262)$ & $<0.001$ & 0.405 & $(0.241-0.656)$ & 0.001 \\
\hline $\begin{array}{l}\text { Fuhrman grade } \\
\text { I-II } \\
\text { III-IV }\end{array}$ & 0.510 & $(0.286-0.842)$ & 0.008 & 0.368 & $(0.121-1.109)$ & 0.083 \\
\hline
\end{tabular}



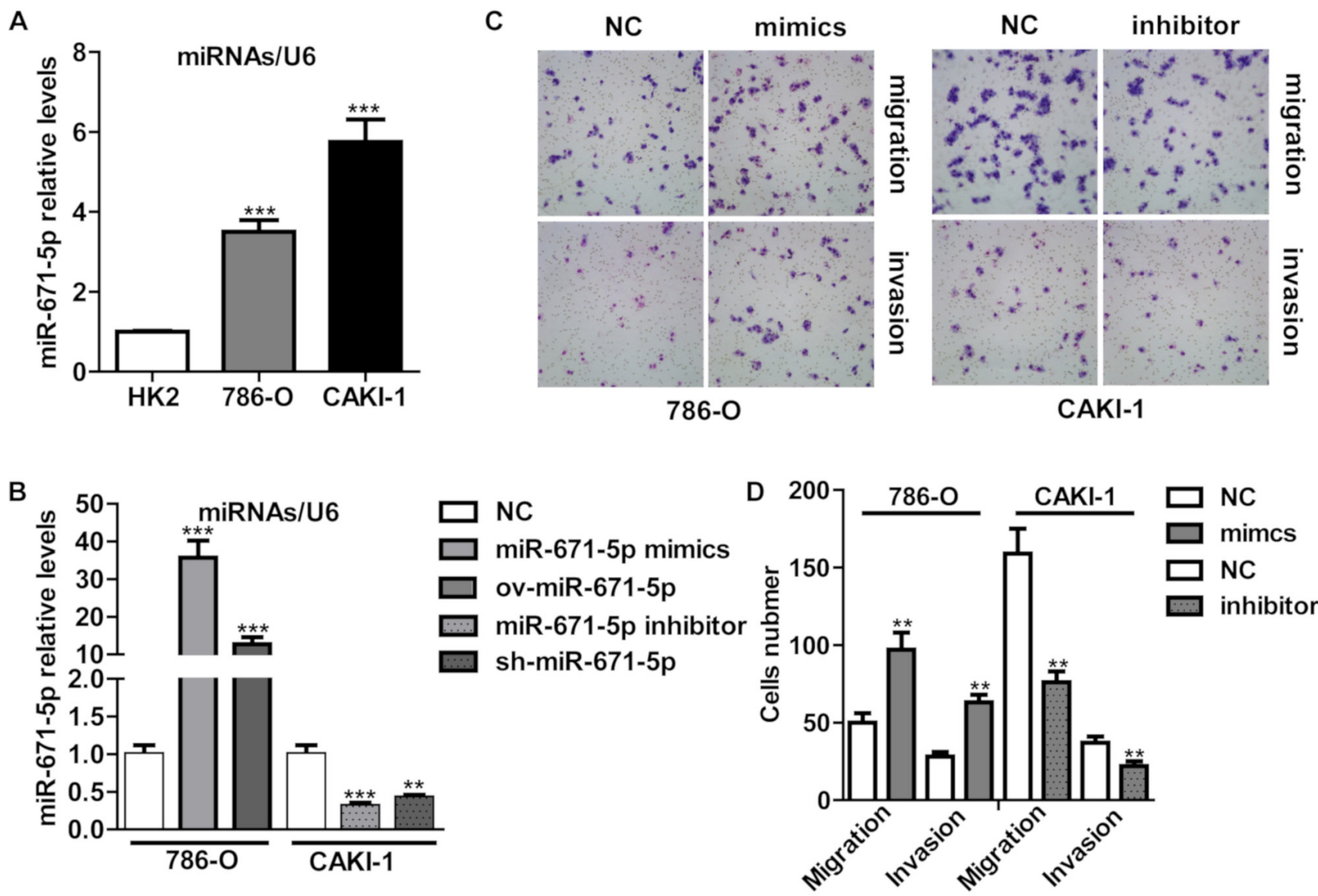

E
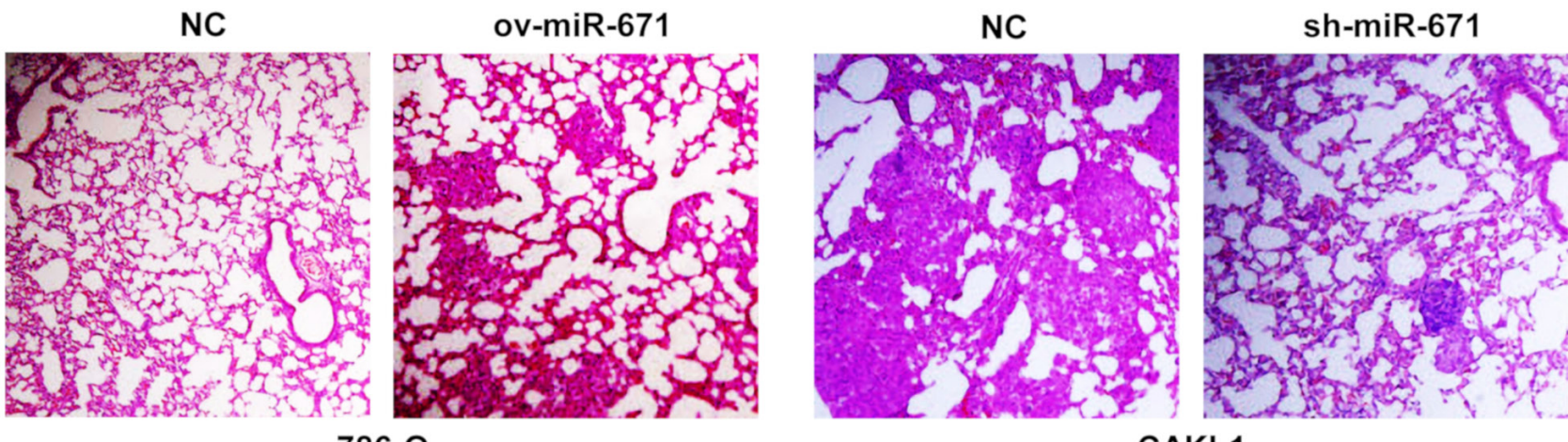

786-O

CAKI-1

Figure 2. miR-671-5p promotes migration and invasion of ccRCC cells. A) The endogenous expression levels of miR-671-5p in HK2, 786-O and CAKI-1 cells. B) Relative miR-671-5p expression in 786-O cells with miR-671-5p overexpression, CAKI-1 cells with miR-671-5p knockdown, and their control cells. C-D) Migration and invasion assays of miR-671-5p-overexpressed 786-O cells, miR-671-5p-silenced CAKI-1 cells and their control cells (scale bar: $10 \mu \mathrm{m}$ ). E) Microscopic pathology of lungs with H\&E staining from the xenograft models (scale bar: $50 \mu \mathrm{m}$ ). ${ }^{\star *} \mathrm{p}<0.01,{ }^{* * *} \mathrm{p}<0.001$.

and transfection of miR-671-5p mimics impaired the luciferase activity in CAKI-1 cells. Furthermore, these effects on luciferase activity were abrogated when the cells were co-transfected with a mutated APC reporter (Figure 3D). Transwell and boyden assays further demonstrated that APC regulated the stimulating effect of miR-671-5p on metastasis of ccRCC cells (Figures 3E, 3F). Subsequently, TOP/FOP analysis and western blot analysis showed that miR-671-5p targeted APC to facilitate the activation of Wnt signaling and to activate the EMT signals, including a decrease of $\mathrm{E}$-cadherin expression and an increase of $\mathrm{N}$-cadherin and Vimentin expression (Figures 3G, 3H).

HMGA1 enhances miR-671-5p expression by binding to its promoter region. In order to identify the transcriptional 
regulatory mechanisms of miR-671-5p, Genomatix bioinformatics software was applied to analyze a $2-\mathrm{kb}$ region upstream of the transcription start site of miR-671-5p. One HMGA1 binding motif at -728 to -722 was identified inside the miR-671-5p promoter region. Furthermore, QPCR analysis confirmed that pre-miR-671 and mature miR-671-5p levels were both increased in HMGA1-overexpressed 786-O cells and decreased in HMGA1-silenced CAKI-1 cells, suggesting that HMGA1 is an upstream regulator of miR-671-5p (Figure 3I). Chromatin immunoprecipitation (ChIP) assay further confirmed that the predicted HMGA1 binding site was functional in the promoter of miR-671-5p in 786-O and CAKI-1 cells (Figure 3J).

Correlations between miR-671-5p, HMGA1, APC expression and clinicopathological characteristics in ccRCC. To further explore the clinical significance of miR-671-5p, HMGA1 and APC in ccRCC, we performed
Table 4. Incidence of lung metastasis in mice.

\begin{tabular}{lc}
\hline & Lung metastasis \\
\hline mice injected with 786-O cells & $5 / 10$ \\
mice injected with 786-O-ov-miR-671-5p cells & $9 / 10$ \\
mice injected with CAKI-1 cells & $7 / 10$ \\
mice injected with CAKI-1-sh-miR-671-5p cells & $3 / 10$ \\
\hline
\end{tabular}

bioinformatics analysis based on the TCGA database. Consistent with the experimental findings, HMGA1 was positively correlated with miR-671 expression; however, miR-671 was negatively correlated with APC expression (Figures 4A, 4B). Moreover, survival analyses indicated that HMGA1 expression was positively correlated with a poor prognosis of ccRCC patients (Figure 4C), however, APC levels was negatively correlated with a poor prognosis of ccRCC patients (Figure 4D).
A

APC 3' UTR 5' ...UCUGUUGUUUUCCCA GGCUUCCA ...

miR-671-5p 3' GAGGUCGGGGAGGUCC $\overline{C G A A G G}$

B
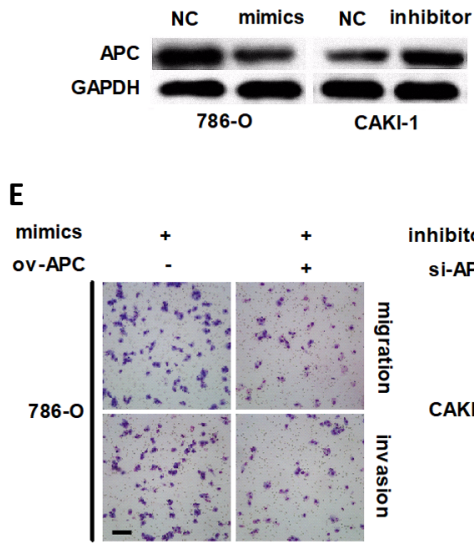

G

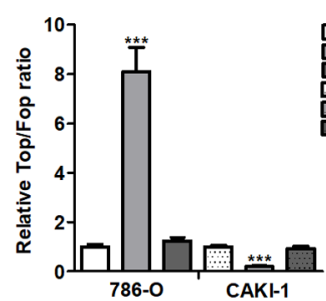
mimics mimics inhibitor
inhibitor+si-APC
C

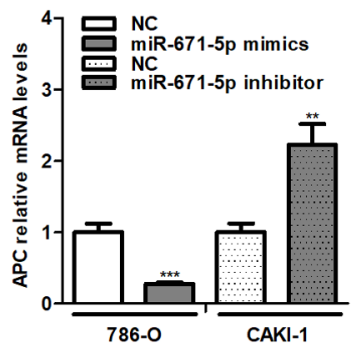

D

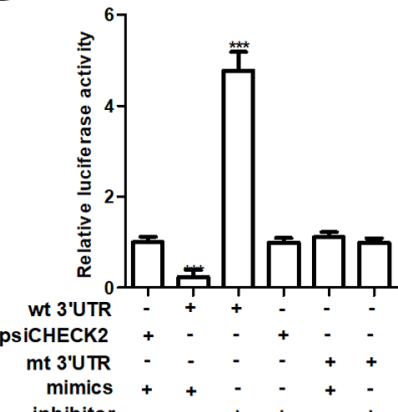

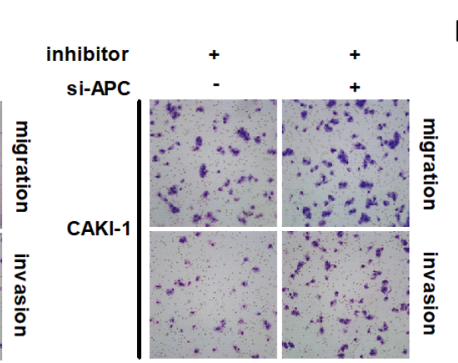

$\mathbf{F}$

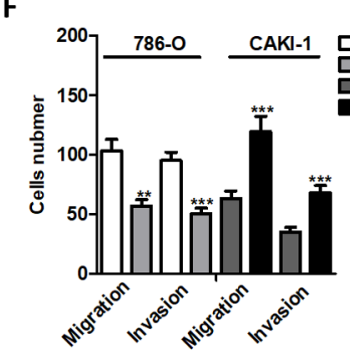

H

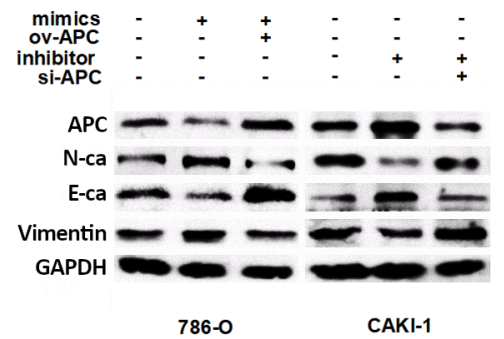

CAKI-1

inhibitor - - + + - +

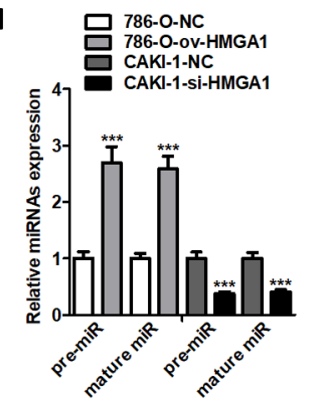

J

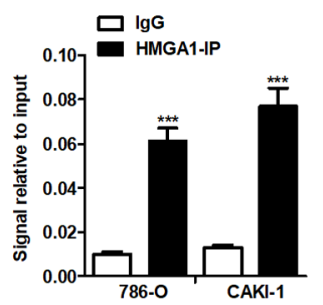

Figure 3. HMGA1-mediated miR-671-5p directly targets APC to activate Wnt signaling. A) miR-671-5p and its putative binding sequences in the 3'UTR of APC. B) Western blot analysis of APC protein levels in miR-671-5p-overexpressed 786-O cells, miR-671-5p-silenced CAKI-1 cells and their control cells. C) QPCR analysis of APC mRNA levels in miR-671-5p-overexpressed 786-O cells, miR-671-5p-silenced CAKI-1 cells and their control cells. D) Luciferase reporter assays were performed to explore whether miR-671-5p directly targeted the 3'UTR of APC in CAKI-1 cells. E-F) The effect of miR-671-5p and APC on migration and invasion of 786-O and CAKI-1 cells (scale bar: $10 \mu \mathrm{m}$ ). G) TOP/FOP analysis of the effect of miR-671-5p and APC on the activity of Wnt signaling. $\mathrm{H}$ ) The effect of miR-671-5p and APC on E-ca, N-ca, and Vimentin protein levels in 786-O and CAKI-1 cells. I) QPCR analysis of pre-miR-671 and mature miR-671-5p expression in HMGA1-overexpressed 786-O cells, HMGA1-silenced CAKI-1 cells and their control cells. J) ChIP analysis of HMGA1 binding to the miR-671-5p promoter region in 786-O and CAKI-1 cells. ${ }^{* *} \mathrm{p}<0.01,{ }^{* * *} \mathrm{p}<0.001$. 

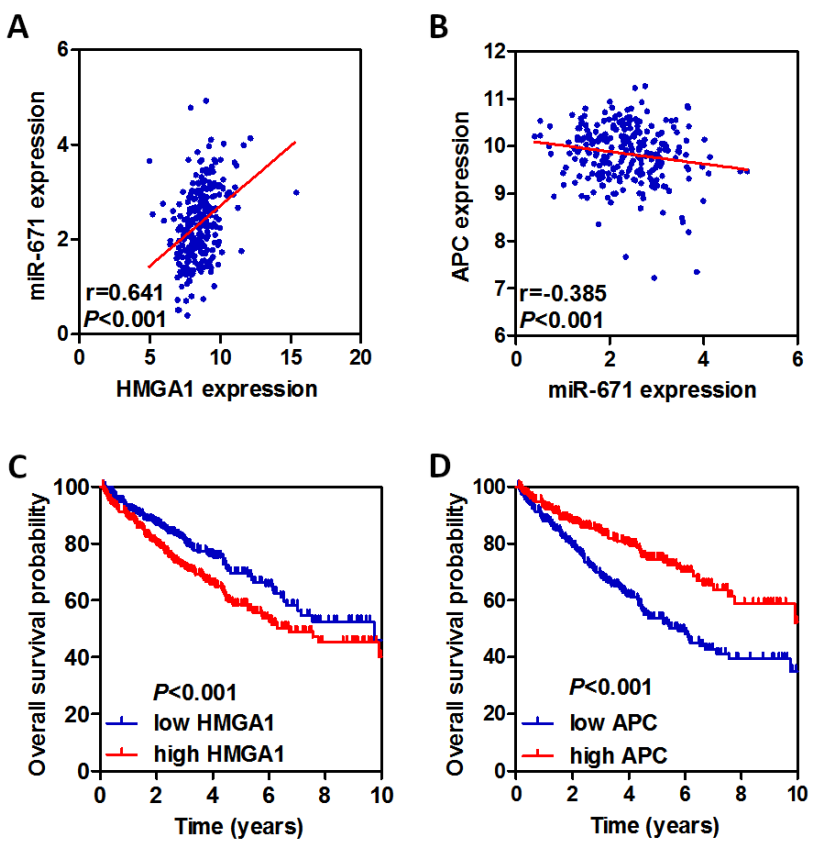

Figure 4. Correlations between miR-671-5p, HMGA1, APC expression and clinicopathological characteristics in ccRCC. A) The correlation between HMGA1 and miR-671-5p. B) The correlation between miR-671-5p and APC expression. C) Kaplan-Meier survival analysis of ccRCC patients with low and high HMGA1 expression based on TCGA KIRC mRNA-seq data. D) Kaplan-Meier survival analysis of ccRCC patients with low and high APC expression based on TCGA KIRC mRNA-seq data.

\section{Discussion}

miRNAs can act as oncomiRs or tumor suppressors in ccRCC [9-11]. However, the effects and molecular mechanisms of miR-671-5p in ccRCC progression have yet to be elucidated. In this work, we demonstrated that miR-671-5p was upregulated in ccRCC tissues compared with paracarcinoma tissues and was identified as independent prognostic factor for overall survival in ccRCC patients. miR-671-5p promoted the migration and invasion of ccRCC in vivo and in vitro via facilitating the EMT process, including downregulating E-cadherin and upregulating $\mathrm{N}$-cadherin and vimentin. Mechanistically, miR-671-5p stimulated ccRCC metastasis by directly targeting APC, thus activating Wnt signaling to promote EMT in ccRCC.

The previous studies suggested that miR-671 was an onco-miRNA in non-small-cell lung cancer $[12,13]$ and a tumor suppressor miRNA in gastric cancer and breast cancer $[14,15]$. In present work, we found that miR-671-5p was upregulated in ccRCC tissues compared with paracarcinoma tissues, and was positively associated with overall survival of ccRCC patients. Our findings are consistent with the previous report that suggested that miR-671was significantly associated with overall survival in clear cell renal cell carcinoma patients [16]. It is reported that miR-671 is involved in regulating cell proliferation, apoptosis and metastasis in cancers. Consistently, we demonstrated that miR-671-5p facilitated EMT process to promote ccRCC metastasis and functioned as an oncomiR. APC is a classical tumor suppressor in human cancers. APC can facilitate ubiquitin-mediated proteolysis of $\beta$-catenin, thus inactivating Wnt signaling [17]. Here, we showed that miR-671-5p directly targeted APC to downregulate its expression, activating Wnt signaling in ccRCC. The activation of Wnt signaling is responsible for epithelial-mesenchymal transition in cancers [18]. We demonstrated that miR-671-5p directly targeted APC to activate Wnt signaling that facilitated EMT and ccRCC metastasis.

Numerous studies have established a clear link and functional role for HMGA1 overexpression and high-grade, aggressive cancers, from its initial discovery in the extraordinarily proliferative HeLa human cervical cancer cells to more recent identification of HMGA1 as a key transcription factor enriched in poorly differentiated cancers with adverse outcomes [19]. In renal cancer, HMGA1 knockdown was shown to suppress colony formation, induce apoptosis and inhibit invasion and migration in ccRCC cells. HMGA1 was identified as a potential target for novel therapeutic modalities for metastatic renal cell carcinoma [20]. In this study, we suggested that HMGA1 transcriptionally enhanced miR-671-5p expression. The miR-671-5p-mediated Wnt signaling was responsible for the specific mechanism that HMGA1 activated Wnt signaling [21]. The newly identified signaling further clarified the roles of HMGA1/miR-671-5p/ APC in ccRCC metastasis.

Bioinformatics analysis and in situ hybridization assays verified that miR-671-5p functioned as an onco-miRNA to promote ccRCC metastasis and served as an independent prognostic factor for overall survival in ccRCC patients. Intriguingly, gene set enrichment analysis of TCGA ccRCC data confirmed that miR-671-5p positively participate in the modulation of EMT process. The positive correlation between HMGA1 and miR-671-5p and the negative correlation between miR-671-5p and APC supported the regulation of HMGA1/miR-671-5p/APC signaling. The survival analysis of HMGA1, miR-671-5p and APC suggested their crucial roles in ccRCC progression.

Taken together, we demonstrated that miR-671-5p acted as an oncogene to facilitate EMT process in ccRCC, promoting cancer metastasis in vivo and in vitro. Meanwhile, miR-671-5p directly targeted the classical tumor suppressor APC to augment Wnt signaling. Interestingly, the oncogene HMGA1 transcriptionally enhanced miR-671-5p expression to form HMGA1/miR-671-5p/APC signaling, thus inducing EMT process. miR-671-5p, the potential factor for the clinical diagnosis or prognosis of ccRCC, could be an effective anticancer target for ccRCC treatment.

Acknowledgements: The authors would like to compliment staff of the Pathology Department for providing a good environment and facilities to finally complete this project. 


\section{References}

[1] ZNAOR A, LORTET-TIEULENT J, LAVERSANNE M, JEMAL A, BRAY F. International variations and trends in renal cell carcinoma incidence and mortality. Eur Urol 2015; 67: 519-530. 10.1016/j.eururo.2014.10.002

[2] HSIEH JJ, PURDUE MP, SIGNORETTI S, SWANTON C, ALBIGES L et al. Renal cell carcinoma. Nat Rev Dis Primers 2017; 3: 17009. https://doi.org/10.1038/nrdp.2017.9

[3] CHAFFER CL, SAN JUAN BP, LIM E, WEINBERG RA. EMT, cell plasticity and metastasis. Cancer Metastasis Rev 2016; 35: 645-654. https://doi.org/10.1007/s10555-0169648-7

[4] YANG J, WEINBERG RA. Epithelial-mesenchymal transition: at the crossroads of development and tumor metastasis. Dev Cell 2008; 14: 818-829. https://doi.org/10.1016/j.devcel.2008.05.009

[5] THIERY JP. Epithelial-mesenchymal transitions in tumour progression. Nat Rev Cancer 2002; 2: 442-454. https://doi. org/10.1038/nrc822

[6] Liu W, Zhang B, Chen G, Wu W, Zhou L et al. Targeting miR-21 with Sophocarpine Inhibits Tumor Progression and Reverses Epithelial-Mesenchymal Transition in Head and Neck Cancer. Mol Ther 2017; 25: 2129-2139. https://doi. org/10.1016/j.ymthe.2017.05.008

[7] ROKAVEC M, HORST D,HERMEKING H. Cellular Model of Colon Cancer Progression Reveals Signatures of mRNAs, miRNA, lncRNAs, and Epigenetic Modifications Associated with Metastasis. Cancer Res 2017; 77: 1854-1867. https:// doi.org/10.1158/0008-5472.CAN-16-3236

[8] MUSAVI SHENAS MH, EGHBAL-FARD S, MEHRISOFIANI V, ABD YAZDANI N, RAHBAR FARZAM O et al. MicroRNAs and signaling networks involved in epithelial-mesenchymal transition. J Cell Physiol 2019; 234: 5775-5785. https://doi.org/10.1002/jcp.27489

[9] YANG L, ZHAO G, WANG F, LI C,WANG X. HypoxiaRegulated miR-146a Targets Cell Adhesion Molecule 2 to Promote Proliferation, Migration, and Invasion of Clear Cell Renal Cell Carcinoma. Cell Physiol Biochem 2018; 49: 920931. https://doi.org/10.1159/000493224

[10] WANG Y, DONG D, JIANG S, ZHANG E, ZHENG W et al. miR-216b Post-Transcriptionally Downregulates Oncogene KRAS and Inhibits Cell Proliferation and Invasion in Clear Cell Renal Cell Carcinoma. Cell Physiol Biochem 2018; 49: 1755-1765. https://doi.org/10.1159/000493621
[11] HuangH,XiongG,ShenP,CaoZ,ZhengLetal.MicroRNA-1285 inhibits malignant biological behaviors of human pancreatic cancer cells by negative regulation of YAP1. Neoplasma 2017; 64: 358-366. https://doi.org/10.4149/neo_2017_306

[12] YU Y, WANG Z, SUN D, ZHOU X, WEI X, et al. miR-671 promotes prostate cancer cell proliferation by targeting tumor suppressor SOX6. Eur J Pharmacol 2018; 823: 65-71. https://doi.org/10.1016/j.ejphar.2018.01.016

[13] BARBAGALLO D, CONDORELLI A, RAGUSA M, SALITO L, SAMMITO M et al. Dysregulated miR-671-5p / CDR1-AS / CDR1 / VSNL1 axis is involved in glioblastoma multiforme. Oncotarget 2016; 7: 4746-4759. https://doi.org/10.18632/ oncotarget.6621

[14] QIU T, WANG K, LI X, JIN J. miR-671-5p inhibits gastric cancer cell proliferation and promotes cell apoptosis by targeting URGCP. Exp Ther Med 2018; 16: 4753-4758. https:// doi.org/10.3892/etm.2018.6813

[15] TAN X, FU Y, CHEN L, LEE W, LAI Y et al. miR-671-5p inhibits epithelial-to-mesenchymal transition by downregulating FOXM1 expression in breast cancer. Oncotarget 2016; 7: 293-307. https://doi.org/10.18632/oncotarget.6344

[16] GE YZ, XU LW, ZHOU CC, LU TZ, YAO WT et al. A BAP1 Mutation-specific MicroRNA Signature Predicts Clinical Outcomes in Clear Cell Renal Cell Carcinoma Patients with Wild-type BAP1. J Cancer 2017; 8: 2643-2652. https://doi. org/10.7150/jca.20234

[17] NIEHRS C. The complex world of WNT receptor signalling. Nat Rev Mol Cell Biol 2012; 13: 767-779. https://doi. org/10.1038/nrm3470

[18] Yue B, Liu C, Sun H, Liu M, Song C et al. A Positive FeedForward Loop between LncRNA-CYTOR and Wnt/ $\beta$ Catenin Signaling Promotes Metastasis of Colon Cancer. Mol Ther 2018; 26: 1287-1298. https://doi.org/10.1016/j. ymthe.2018.02.024

[19] SUMTER TF, XIAN L, HUSO T, KOO M, CHANG YT et al. The High Mobility Group A1 (HMGA1) Transcriptome in Cancer and Development. Curr Mol Med 2016; 16: 353-393. https://doi.org/10.2174/1566524016666160316152147

[20] TAKAHA N, SOWA Y, TAKEUCHI I, HONGO F, KAWAUCHI A et al. Expression and role of HMGA1 in renal cell carcinoma. J Urol 2012; 187: 2215-2222. https://doi. org/10.1016/j.juro.2012.01.069

[21] RESAR L, CHIA L,XIAN L. Lessons from the Crypt: HMGA1-Amping up Wnt for Stem Cells and Tumor Progression. Cancer Res 2018; 78: 1890-1897. https://doi. org/10.1158/0008-5472.CAN-17-3045 